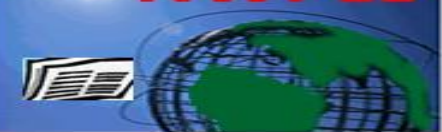

American Journal of Biomedical Sciences

ISSN: 1937-9080

nwpii.com/ajbms

\title{
An Overview on Relation between the Bradykinin System and Hypertension and Diabetes
}

\author{
J. N. Sharma*
}

Department of Applied Therapeutics, Faculty of Pharmacy, Health Sciences Centre, Kuwait University, Kuwait

*Corresponding Author:

J. N. Sharma

Department of Applied Therapeutics

Faculty of Pharmacy, Health Sciences Centre

Kuwait University, P.O. Box 24923

Safat 13110, Kuwait

Email: j.n.sharma@hsc.edu.kw

Received: 3 May 2009; | Revised: 11 May 2009; | Accepted: 15 May 2009

\section{Summary}

It is proposed here that a deficiency of BK might be a significant factor in the pathophysiology of hypertension and diabetes. In this regard, it is suggested that the role of renal BK is to excrete the excess sodium. Therefore, a reduction in the generation of renal BK may be the cause for the development of hypertension as a result of the accumulation of sodium in the body. Thus, the development of a compound having renal kallikrein-like activity may serve the purpose of excreting excessive sodium from the kidney in the treatment of hypertension. Transgenic mice over-expressing renal tissue kallikrein were hypotensive and that administration of aprotinin, a tissue kallikrein inhibitor, restored the BP of the transgenic mice. Recently, it has been proposed that tissue kallikrein gene delivery into various hypertensive models exhibits protection, such as reduction in high blood pressure, attenuation of cardiac hypertrophy, inhibition of renal damage and stenosis. This may indicate the future therapeutic aspect of kallikrein gene therapy for hypertension, cardiovascular and renal pathology.

Keywords: bradykinin system; diabetes; hypertension; overview.

\section{Introduction}

Cardiovascular diseases are the most common cause of mortality worldwide. Hypertension and diabetes are the two major risk factors in the development of cardiac hypertrophy, ischemic heart disease and cardiac failure. Previous studies have indicated altered activities of the bradykinin (BK)-generating components in hypertension and diabetes [1]. Bradykinin is a pharmacologically active polypeptide that can promote both cardiovascular and renal function, for example, 
vasodilation, natriuresis, diuresis and release of nitric oxide (NO) [2, 3]. In addition, B2 kinin receptors are present in the cardiac endothelial cells which may enhance the biosynthesis and release of NO. Sharma and Uma [4] demonstrated that reduced tissue kallikrein levels may be associated with the development of high blood pressure in spontaneously hypertensive and diabetic rats (SHR). The BK may produce their pharmacological effects via NO and cyclic GMP release [5].

Furthermore, it has been established that the kinin has cardioprotective action in myocardial ischemia and can prevent left ventricular hypertrophy [3]. Also, transgenic mice carrying tissue kallikrein gene and over expressing tissue kallikrein had reduced blood pressure [6]. NO synthase and renal tissue kallikrein are both involved in blood pressure regulation $[7,8]$

\section{The bradykinin system}

Bradykinin (BK) is one of kinins, a pharmacologically active polypeptide, which is released in the tissues and body fluids as a result of enzymatic action of kallikreins on kininogens. The kinins are the BK (Arg-Pro-Pro-gly-Phe-SerPro-Phe-Arg), Kallidin (Lys-Arg-Pro-Pro-GlyPhe-Ser-Pro-Phe-Arg) and methionyl-lysyl-BK (Met-Lys-Arg-Pro-Pro-Gly-Phe-Arg). Kallidin and methionyl-lysyl-BK are converted into BK by aminopeptidases present in plasma and urine. BK is rapidly ( $<15 \mathrm{sec}$ ) inactivated by circulating kinases (Sharma 2006). Once BK is released in the blood and body fluids, it may act on two BK receptors known as B1 and B2.

Kininogens are multifunctional proteins derived mainly from alpha- 2 globulin. In humans the two forms of kininogens are high molecular weight kininogen (HMWK) and low molecular weight kininogen (LMWK). These kininogens vary from each other in molecular weight, susceptibility to plasma and tissue kallikreins and in their physiological properties [1]. They are synthesized in the liver and circulate in the plasma and other body fluids.

There are two forms of kallikreins known as tissue kallikrein and plasma kallikrein. Tissue kallikrein is found in various organs such as the kidney, heart and synovial tissue. The tissue kallikrein is synthesized in the cell as a precursor and converted into the active form by the cleavage of an amino terminal peptide [9]. Active tissue kallikrein acts on LMWK to release kallidin. Tissue and plasma kallikreins differ from one another in molecular weight, biological functions, physicochemical and immunological properties. The plasma kallikrein is present in circulation in an inactive form, which is known as prekallikrein or Fletcher factor. This inactive prekallikrein is converted to active kallikrein by activated Hageman factor (XIIa). In addition, plasma kallikrein is able to convert inactive factor XII to XIIa by positive feedback reaction [9]. The plasma prekallikrein and HMWK are present together in a complex form. Factor XIIa and factor XI circulate with HMWK in bound form. In this way, factor XI can be converted into XIIa for the participation in the intrinsic coagulation cascade. In immunological reactions, the tissue proteoglycan and mast cell heparin might act as an initiating surface for initial activation of the Hageman factor [10]. It seems that the kinins may be generated in parallel with the formation of thrombin at inflammatory sites, since inactive plasma kallikrein can be activated by coagulant Hageman factor. The tissue kallikrein multigene family comprises closely related cluster of genes that vary in number between the different mammalian species: 24 genes have been identified in the mouse, 20 in the rat, 3 in humans and 3 in the hamster $[11,1]$.

Several restriction fragment length polymorphisms (RFLP) have been mapped in tissue kallikrein gene and their regulatory regions in SHR[12]. These findings may reflect a possible difference in the tissue kallikrein gene locus between SHR and normotensive Wistar-Kyoto rats (WKYR). A tissue kallikrein RFLP has been indicated to co segregate with high blood pressure (BP) in the F2 offspring of SHR and normotensive Brown Norway rat crosses [11]. This finding strongly suggests a possibility of a close linkage between the kallikrein gene locus and the hypertensive phenotype of SHR.

The kininases, kinin inactivating enzymes, are present in the plasma, endothelial cells and in the tissues to regulate the physiological functions 
of the kinins in the body. These are known as kininase I, kininase II or angiotensin converting enzyme (ACE) and encephalinase. In plasma, kininase I cleaves the $\mathrm{C}$-terminal argentine of $\mathrm{BK}$ to form des-Arg9-BK. Kininase II causes inactivation of $\mathrm{BK}$ by releasing pentapeptide (Arg-Pro-Pro-gly-Pheo and tripeptide (Ser-ProPhe) fragments. Figure 1 shows the formation of BK.

Interaction between the kinins and their specific receptors can lead to activation of several second messenger systems. The BK receptor stimulation of the intact cells or in tissues appears to initiate the second messenger pathways, such as the arachidonic acid products and the activation of calcium sensitive systems [13]. The elevation of cellular inositol phosphates by BK involves Gprotein coupled activation of phospholipase A2 and $\mathrm{C}$ that are used in the synthesis of eicosanoids
[14]. It is of interest that indomethacin, a cyclooxygenase inhibitor, was able to cause potentiation of BK-induced contractions in guinea pig tracheal smooth muscle preparations [15]. These findings may suggest that there could be non-eicosanoid pathways for the cellular and molecular actions of BK. Furthermore, it is known that BK significantly stimulates phosphoinositide hydrolysis in guinea pig ileum longitudinal muscle that may result in elevation of cytosolic calcium ion levels to induce contractile responses. Schini et al. [16] demonstrated that the B2 receptor stimulation causes production of cyclic GMP in cultured porcine aortic endothelial cells. The formation of cyclic GMP may be an important step for the biological actions as well as release of NO evoked by BK in the endothelial cells and in the vascular smooth muscles.

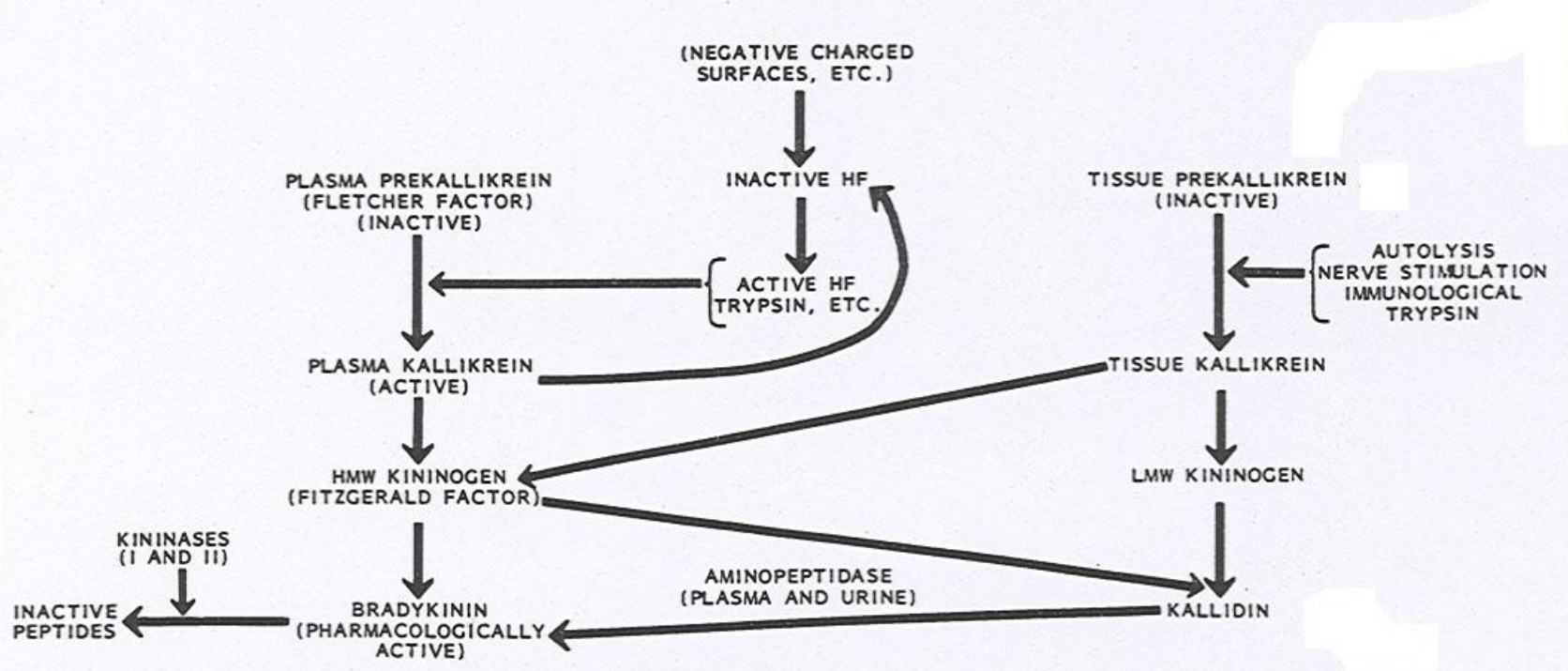

Fig. 1. Scheme of the components of the kinin-forming and inactivating system.

\section{Hypertension and the kinin system}

Hypertension is a major risk factor in the development of cardiovascular diseases, such as coronary heart disease, congestive heart failure and peripheral vascular \& renal diseases. There is ample evidence documenting the role of $\mathrm{BK}$ in the pathogenesis of hypertension $[17,2]$. The pharmacological actions of $\mathrm{BK}$ in regulation of systemic BP are vasodilation in most areas of circulation, reduction in total peripheral vascular resistance and regulation of sodium excretion from the kidney [18]. The role of KKS in hypertension was established by Margolius and co-investigators [19-21] with the observations that urinary kallikrein excretion is significantly reduced in hypertensive patients and hypertensive rats. This led to the suggestion that reduced urinary kallikrein excretion might result from a reduction in kinin generation in hypertensive 
situations. Research on the systemic changes in the kallikrein has provided further insight regarding the mechanisms of various hypertensive conditions. In this connection, it is known that kininogen levels and kinin-forming factors are reduced in essential and malignant hypertension $[22,23]$. It may be that a deficiency of plasma HMWK due to a decrease in liver synthesis (which decreases kinin production) occurs in an individual who develops hypertension after mild exercise [24]. It is proposed here that a deficiency in BK might be a significant factor in the pathophysiology of hypertension. In this regard, it is suggested that the role of renal $\mathrm{BK}$ is to excrete the excess sodium. Therefore, a reduction in the generation of renal BK may be the cause of the development of hypertension as a result of the accumulation of sodium in the body [2]. Thus, the development of a compound having renal kallikrein-like activity may serve the purpose of excreting excessive sodium from the kidney in the treatment of hypertension. Also it has been demonstrated that transgenic mice overexpressing renal tissue kallikrein were hypotensive and that administration of aprotinin, a tissue kallikrein inhibitor, restored the BP of the transgenic mice [6]. We have shown the suppression of hypotensive responses of ACEIs by aprotinin in SHR [25]. These findings highlight a role of tissue kallikrein in the regulation of BP. Recently, it has been proposed that tissue kallikrein gene delivery into various hypertensive models exhibits protection, such as reduction in high BP, attenuation of cardiac hypertrophy, inhibition of renal damage and stenosis [26-29]. This may indicate the future therapeutics aspect of kallikrein gene therapy for cardiovascular and renal pathology.

ACEIs are currently used in the treatment of both clinical and experimental hypertension [30,31]. Kininase II inhibitors could lower BP by inhibiting the biodegradation of kinin as well as blocking the formation of angiotensin II (AgII). A calcium-channel blocker, nifedipine, used in treatment of patients with essential hypertension can normalize the reduced urinary kallikrein excretion [32]. Smith et al. [33] have suggested that women with reduced activity of the renal BK may be at increased risk of developing pregnancy-induced hypertension. Previous study has demonstrated that urinary kallikrein excretion was found to be diminished in family members at risk for hereditary hypertension and that urinary kallikrein may be one of the major genetic markers associated with family history of hypertension [34].

Left ventricular hypertrophy is regarded as an independent risk factor in hypertensive patients in inducing cardiac abnormalities. BK can counter the development of $\mathrm{LVH}$ in rats with hypertension produced by aortic banding $[35,36]$. This antihypertrophic effect of $\mathrm{BK}$ was abolished by the $\mathrm{B}_{2}$ receptor antagonist treatment as well as by NO inhibitor. Thus, the BK has a role in protecting the heart against developing $\mathrm{LVH}$ by releasing NO in this model of hypertension induced by aortic banding. In this regard, we have for the first time demonstrated that the lack of the cardiac KKS could be responsible for the induction of $\mathrm{LVH}$ in SHR and SHR with diabetes [37, 38]. It is suggested that the reduced cardiac tissue kallikrein and cardiac kininogen may be responsible for reduced $\mathrm{BK}$ generation in the heart. Therefore deficient components of the BK system in the heart may be responsible for inducing excessive hypertrophy and myocardial dysfunction in cases of hypertension. It is highly desirable to develop stable compounds of BK to evaluate their efficacy and potency in cases of cardiac failure, cardiac ischemia and hypertension.

It is the generally accepted view that the BKinduced $\mathrm{BP}$ lowering effect is mediated by the $\mathrm{B}_{2}$ receptor, but $B_{1}$ might also be involved under special situations [39]. It has been demonstrated that a $B_{2}$ receptor antagonist (B5630) can generally abolish the hypotensive effects of BK as well as captopril, an ACEI [40]. This led to the proposal that the hypotensive action of ACE inhibitors might be due to the activation of $\mathrm{B}_{2}$ receptor The accumulation of $\mathrm{BK}$ after treatment with ACEIs with subsequent release of NO, PGs and $\mathrm{PGI}_{2}$ could account for additional mediators released in the process of anti-hypertensive action of these drugs in hypertensive patients. 


\section{Diabetes and the kinin system}

Several investigators [41-45] have reported alterations of the renal KKS in the diabetic state. Insulin- treated moderately hyperglycemic diabetic rats and patients with diabetes mellitus have been reported to show increased urinary kallikrein and BK excretion [42]. These findings suggest that alterations in the kinin-forming components may be the indicator of vascular disease in type 1 diabetics. The renal hyper filtration in diabetic rats was reduced after pretreatment with aprotinin, a tissue kallikrein inhibitor, suggesting a role of $\mathrm{KKS}$ in diabetic state of increased glomerular hemodynamics [46]. In addition, Vieira et al. [47] demonstrated the renal conversion of T-kinin (present in the rats) to BK. The conversion of T-kinin, which is the main kinin in inflammation in rats, could be an important alternative pathway for the generation of renal $\mathrm{BK}$ in diabetic rats. On the other hand, the metabolism of $\mathrm{BK}$ might be impaired and it has to be shown whether changes in the activity of kininases could lead to an increased urinary BK excretion under diabetic conditions. In our earlier studies, we observed the reduction in cardiac and plasma kallikrein and kininogen concentrations in hypertensive and diabetic rats [37]. These studies suggested that the development of left ventricular hypertrophy (LVH) and high blood pressure in these diabetic rats could be the reflection of hypoactivity of the KKS. These research findings were indeed supported by the fact that the reduced synthesis of the myocardial tissue kallikrein implies a reduced capacity to generate $\mathrm{BK}$ in diabetic rats $[48,43,49]$. It can be postulated therefore that alterations of the KKS may contribute to the cardiac dysfunction in diabetes mellitus in human patients. Furthermore, it is suggested that the treatment with the KKS components in diabetic conditions may reverse the myocardial abnormalities observed in diabetic patients. Recently, it has been reported that high plasma prekallikrein activity may serve as a marker for the diabetic hypertensive nephropathy [43], which may be the marker of vascular disease in diabetic patients. It has been recently pointed out that cardioprotective effects of the KKS in the diabetic heart suggest that the stimulation of the
KKS might open new avenues for the treatment of diabetic cardiopathy due to down regulation of kinins inactivating enzymes [50,10,51]. Also, BK 2 receptor activation may contribute to the development of diabetic nephropathy [52]. On the other hand, kallikrein gene delivery improves serum glucose and lipid profile and cardiac function in experimental diabetics [53]. Recently, it has been suggested that BK system may be a therapeutic target for preventing and treating diabetic nephropathy [54].

\section{References}

1. Leeb-Lundberg, L.M.F.; Marceau,F.; MullerEsterl,W.; Pettibone, D.J.; Zuraw, B.L. International Union of Pharmacology. XLV. Classification of the kinin receptor family: From molecular mechanisms to Pathophysiological consequences, Pharmacological Review 2005, 57, 27-77.

2. Katori, M.; Majima, M. A missing link between a high salt intake and blood pressure increase, Journal of Pharmacological Sciences, 2006, 100, 370-390.

3. Linz, W.; Wiemer, G.; Scholkens, B. A. Cardioprotective actions of bradykinin in myocardial ischemia and left ventricular hypertrophy, Brazilin Journal of Medical and Biological Research, 1994, 8, 1949-54.

4. Sharma. J. N.; Uma. K. Cardiac kallikrein in hypertensive and normotensive rats with and without diabetes, Immunopharmacology, 1996, 33, 341-343.

5. Carretero, O.A. Vascular remodeling and the kallikrein-kinin system Journal of Clinical Investigation, 2005, 115, 588-591.

6. Wang, C.; Chao, L.; Chao, J. Human tissue kallikrein induces hypotension in transgenic mice, Hypertension, 1994, 23, 236-243.

7. Marcondess, S.; Antunes, E. The plasma and tissue kininogen-kallkein-kinin system: role in the cardiovascular system, Current Medicinal Chemistry, 2005, 3, 33-44.

8. Sharma, J. N.; Uma, K.; Noor, A.R. Blood pressure regulation by the kallikrein-kinin system, General Pharmacology, 1996, 27: 5563. 
9. Moreau, M. E., Garbacki, N., Molinaro, G., Brown, N. J., Marceau, F. and Adam, A. (2005). The kallikrein-kinin system: current and future pharmacological targets, Journal of Pharmacological Sciences, 2005, 99: 6-38.

10. Sharma, J. N. The tissue kallikreinkininogem-kinin pathways: role in cardiovascular system, Archives of Medical Research , 2006, 37, 299-306.

11. Pravence, M.; Ken, V.; Kunes, J. Cosegregation of blood pressure with kallikrein gene family polymorphism, Hypertension, 1991, 17: 242-246.

12. Woolly-Miller, C., Chao, J. and Chao, L. Restriction fragment length polymorphism's mapped in spontaneously hypertensive rats using kallikrein probs, Journal of Hypertension, 1989, 7: 865-871.

13. Farmer, S. G. and Burch, R. M. (1992). Biochemical and molecular pharmacology of kinin receptors, Annual Review of Pharmacology and Toxicology, 1992, 32, 511-536.

14. Burch, R. M. Kinin signal transduction: role of phosphoinositides and eicosanoids, Journal of Cardiovascular. Pharmacology, 1990, 15 (Suppl. 6), S44-S46.

15. Akbar, A.; Sharma J. N.; Yusof A. P. M. Potentiation of bradykinin-induced responses in the intact and denuded epithelium of guinea pig tracheal preparations, Tissue Reactions, 1998, XX, 95-100.

16. Schini, V. B., Boulanger, C., Regoli, D., and Vonhoutte, P.M. Bradykinin stimulates the production of cyclicGMP via activation of B2 reeceptors in cultured porcine aortic endothelial cells, Journal of Pharmacology and Experimental Therapeutics , 1990, 43, 1823-1827.

17. Sharma, J. N. Hypertension and bradykinin system, Current Hypertension Report, 2009 in press.

18. de Freitas, F. M.; Farraco, E. Z.; De Azevedo, D. F. General circulatory alterations induced by intravenous infusion of synthetic bradykinin in man, Circulation , 1964, 29, 6670.

19. Margolius, H. S.; Geller, R.; DeJong, W. (1972). Altered urinary kallikrein excretion in rats hypertension, Circulation Research , 1972, 30, 358-362

20. Margolius, H. S.; Geller, R.; Pisano, J. J. Altered urinary kallikrein excretion in human hypertension, Lancet, 1971, 2, 1063-1065.

21. Margolius, H. S.; Horwwitz, D.; Pisano, J. J. Urinary kallikrein excretion in hypertensive man: relationship to sodium intake and sodium-retaining steroids, Circulation Research, 1974, 35, 820-825.

22. Almeida, F. A.; Stella, R. C. R.; Voos, A. (1981). Malignant hypertension: a syndrome associated with low plasma kininogen and kinin potentiating factor, Hypertension , 1981, 3, 46-50.

23. Sharma, J. N.; Zeitlin, I. J. Altered plasma kininogen in clinical hypertension, Lancet, 1981, 1, 125-126.

24. James, F. W. ; Donaldson, V. H. Decreased exercise tolerance and hypertension in severe hereditary deficiency of plasma kininogen, Lancet, 1981, 1, 889.

25. Sharma, J. N.; Amrah, S. S.; Noor, A. R. Suppression of hypotensive responses of captopril and enalapril by kallikrein inhibitor aprotinin in spontaneously hypertensive rats, Pharmacology, 1995, 50, 363-369.

26. Chao, J.; Chao, L. Kallikrein gene therapy in hypertension, cardiovascular and renal diseases, Gene Therapy and Molecular Biology, 1998, 1, 301-308.

27. Chao, J. and Chao L.(2005). Kallikrein-kinin in stroke, cardiovascular and renal disease, Exp Physiol, 90(3): 291-8.

28. Chao, J.; Bledsoe, G.; Yin, H.; Chao, L, (2006). The tissue kallikrein-kinin system protects against cardiovascular and renal diseases and ischemic stroke independently of blood pressure reduction, Biological Chemistry, 2006, 387, 665-75.

29. Chao, J.; Gao, L.; Hagiwara, M.; Sen, B.; Yang, Z. R.; Chao, L. Tissue kallikrein elicits cardioprotection by derect b2 receptor activation independent of kinin formation, Hypertension, 2008, 52, 715-720.

30. Antonacci, M. Angiotensin converting enzyme (ACE) inhibitors, Annual Review of Pharmacology and Toxicology, 1982, 22, 5787. 
31. Silberbauer, K.; Stanek, B.; Temple, H. Acute hypotensive effect of captopril in man modified by prostaglandin synthesis inhibition, British Journal of Clinical Pharmacology, 1982, 14, 87S-93S.

32. Edery, H.; Rosenthal, T.; Amitzur, G. The influence of SQ 20881 on the blood kinin system of renal hypertensive patients, Drug in Experimental and Clinical Research, 1981, VII, 749-756.

33. Smith, C.; Campbell, S.; Albano, J. Urinary kallikrein excretion in normotensive and hypertensive pregnancies: 8 years later, Immunopharmacology , 1999, 44, 177-182.

34. Kailasam, M.T.; Martinez, J.A.; Cervenka, J.H.; Yen, S.S.C.;O'Connor, D.T.; Parmer, R.J. Racial differences in renal kallikrein excretion: Effect of the ovulatory cycle. Kidney International, 1998, 54, 1652-1658.

35. Linz, W., Wiemer, G. and Scholkens, B. A. Bradykinin prevents left ventricular hypertrophy in rats, Journal of Hypertension, 1993, 11 (Suppl. 5), S96-S97

36. Madeddu, P.; Emanueeli, C.; El-Dahr, S. Mechanisms of disease; tiss kallikrein-kinin system in hypetension and vascular remodeling, Nature Clinical Practice and Nephrology, 2007, 3, 208-221.

37. Sharma, J. N.; Uma, K. ; Yusof, A. P. M. Left ventricular hypertrophy and its relation to cardiac kinin-forming system in hypertensive and diabetic rats, International Journal of Cardiology, 1998, 63, 229-235.

38. Sharma, J. N.; Uma, K.; Yusof, A. P. M. Altered cardiac tissue and plasma kininogen levels in hypertensive and diabetic rats, Immunopharmacology, 1999, 43, 129-132.

39. Regoli, D. Neurohumoral regulation of precapillary vessels: the kallikrein-kinin system, Journal of Cardiovascular Pharmacology, 1984; 6 (suppl. 3, S401 S412.

40. Sharma, J. N. Angiotensin Converting Enzyme Inhibitors, DeBrue, A. N.,ED; Nova Science Publishers, Inc., 2009 in press.

41. Jaffa, A. A.; Miller, D.H.; Bailey, G.S.; Chao, J.; Margolius, H.S. Abnormal regulation of renal kallikrein in experimental diabetes,
Journal of Clinical Investigation, 1987, 80, 1651-1659.

42. Jaffa, A. A.; Rust, P.F.; Mayfield, R.K. Kinin, a mediator of diabetes induced glomerular hyper filtration, Diabetes, 1995, 44, 156-160.

43. Jaffa, A. A.; Durazo-Arvizu, R.; Zheng, D.; Lackland, D. T., Srikanth, S.; Garvey, W. T.; Schmaier, A. H. Plasma prekallikrein: a risk marker for hypertension and nephropathy in type 1 diabetes, Diabetes, 2003, 52, 1215-21.

44. Harvey J. N.; Jaffa, A.A.; Margolius, H.S. Renal kallikrein abnormalities of diabetic kidney, Diabetes, 1990, 39, 299-303.

45. Platts, J. K., Meadows, P.; Harvey, J. N. The relationship between urinary kallikrein and glomerular filtration rate (GFR) in type-1 diabetes: studies with lithium, Immunopharmacology, 1996, 33, 351-353.

46. Tschope, G.; Gavriluk, V.; Reinecke, A. Bradykinin excretion is increased in severely hyperglycemic streptozotocin-diabetic rats, Immunopharmacology, 1996, 33, 344-348.

47. Vieira, M.A.R.; Moreira, F.M.; Maack, T. Conversion of T-kinin to bradykinin by the rat kidney, Biochemical Pharmacology, 1994, 47, 1693-1699.

48. Tshope, C.; Reinecke, A.; Seidl, U.; Yu, M.; Gavriluk, V.; Riester, U.; Gohlke, P.; Graf, K.; Bader, M.; Hilgenfeldt, U.; Pesquero, J. B.; Ritz, E.; Unger, T. Functional, biochemical, and molecular investigations of renal kallikrein-kinin system in diabetic rats, American Journal of Physiology, 1999, 277: H2333-2340.

49. Sharma, J. N.; Kesavarao, U . Changes in plasma prekallikrein activity, blood pressure and left ventricular thickness in hypertensive and mormotensive diabetic rats, Methods and Findings in Experimental and Clinical Pharmacology, 2007, 29, 75-78.

50. Spillmann, F.; Van Linthout, S.; Schultheiss, H.P.; Tschope,C. Cardioprotective mechanisms of the kallikrein-kinin system in diabetic cardiopathy, Current Opinion in Nephrology and Hypertension, 2006, 15, 229.

51. Koch, M.; Wendorf, M.; Dendorfer, A.; Wolfrum, S.; Schulze, K.; Spillmann, F.; Schultheiss, H.P.; Tschope, C. Cardiac kinin 
level in experimental diabetes mellitus:role of kinases, American Journal of Physiology Heart Circulation Physiology, 2003, 285, H418-23.

52. Tan,Y.; Wang, B.; Keum, J.S.; Jaffa, A.A. (2005). Mechanisms through which bradykinin promotes glomerular injury in diabetes, American Journal of Physiology Renal Physiology, 2005, 288, F483-92.

53. Montanari, D.; Yin, H.; Dobrzynski, E.; Agata, J.; Yoshida, H.; Chao, J.; Chao, L.
Kallikrein gene delivery improves serum glucose and lipid profiles and cardiac function in streptozotocin-induced diabetic rats, Diabetes, 2005, 54, 1573-80.

54. Riad, A.; Zhuo, J. L.; Schultheiss, H. P.; Tschope, C. The role of the renal kallikreinkinin system in diabetic nephropathy, Current Opinion in Nephrology and Hypertension, 2007, 16, 22-26. 